\title{
Numerical and Experimental Study of a Scale Bus Model in Wind Tunnel
}

\author{
César Alejandro Varela-Boydo and Laura Lilia Castro-Gómez \\ Centro de Investigación en Ingeniería y Ciencias Aplicadas, Instituto de Investigación en Ciencias Básicas y Aplicadas, Universidad \\ Autónoma del Estado de Morelos, Cuernavaca 62209, Morelos, México
}

\begin{abstract}
The main objective of the present study is to determinate which turbulence model can predict more accurately the aerodynamic drag of a bus on scale down wind tunnel testing. In order to achieve this, 1:20 scale models were made by computer and printed with current 3D printing techniques. These models were then putted into the tests in wind tunnel at different free stream velocities; The aerodynamic drag produced during the test was measured. The same experimental conditions were replicated by computer in the ANSYS FLUENT environment and were simulated by a number of different turbulence models. The experimental and numeric results were compared, and as a result of this comparison, the turbulence model that can predict more efficiently the wind tunnel results was found. This models and conditions will then be used to perform simulations of new buses' body modification proposals in order to increase their aerodynamic behaviour.
\end{abstract}

Key words: Bus, wind tunnel, aerodynamic drag, CFD (computational fluid dynamics), turbulence models.

\section{Introduction}

Means of transport are in continuous improvement, while the industrial and technological development never stops and every year transports become faster, more reliable, ecologic and, as a consequence, more efficient. Due to environmental degradation and constant rise of fossil fuels' prices, for the last years, it has been given much more emphasis in diminishing transport means' energetic consumption. Speaking more specifically about land transports, like cars and trucks, it can be found a great amount of scientific articles and patents that show improvements in different parts of the vehicles like: internal combustion engines, electric engines and their batteries, cooling systems, exhaust systems, tribology in all the components, electronics and aerodynamics. Advances in aerodynamics have been focused in reducing the aerodynamic drag, as well as increasing the vehicles stability, trying to make transport faster, safer and cheaper to run. The study proposed in this paper is

Corresponding author: César Alejandro Varela-Boydo, master, research fields: green energies, sustainable transports and turbomachinery. E-mail: c.a.varela.boydo@gmail.com. focused in the passenger bus' aerodynamics and tries to determinate which turbulence models are able to predict more accurately the drag on a 1:20 bus-alike body inside a wind tunnel. This was planning on future body design modifications to improve buses' aerodynamics and eventually creating more fuel efficient designs that could be mass produced.

This paper shows results of numerous CFD (computational fluid dynamics) simulations and scale down wind tunnel testing, which allowed to determinate turbulence models that predict more effectible and with the smallest amount of computer resources and the behaviour of the fluid around a bus-alike body inside a wind tunnel. This work is justified because, in the literature, there exist different published papers with similar objectives but with different conclusions. Every publication was in its own experimental conditions, very specific for every case, and finding a consensus about the better turbulence model to use in automotive CFD simulations was not possible.

There are lots of different kinds of publications about the buses or bus-alike bodies, even when they do 
not come in large numbers. More specifically, the papers about the study or optimization of bus' aerodynamics are quite rare. That brings us one great opportunity to conduct technology developments in the bus' aerodynamics sector. In 1984, Ahmed et al. [1] proposed one bus body interpretation in the shape of a static bluff body, which takes the more important features of a bus body and performed wind tunnel experiments. This body is now called as "Ahmed's body", and the first experiments helped to determinate the drag that was up to the $85 \%$ and produced by a body at high speeds due to pressure drag. Other research groups continued the studies of the Ahmed's body, using it to study among other things, such as the wake behind it and vortex shedding [2]. By a large variety of experimental techniques, they characterized the pressure fields and distribution of air forces, the flow characteristic frequencies, the stream lines and the vortex structures on the tale of the body. All this information together allowed them to prove how complex and difficult to study the flow around bluff bodies is. Different techniques and models have been used, trying to simulate the flow around these bodies, but so far, it has not been possible to find a general solution for these cases. Ahmed's body has been used by different research groups, looking for an efficient way to simulate the flow where the body is immersed [3-6]. Many turbulence models, meshing techniques and simulation platforms have been used to obtain good result for very specific bus or bus-alike body cases.

Passenger buses scale models with similar dimensions to the ones used in this study also have been putted to the test inside wind tunnels and by CFD [7]. They used 1:20 scale bodies and tested them in the wind tunnel to determinate the change in the pressure field on the surface of the bus bodies under cross wind conditions. Many of the techniques [7] used to quantify forces are similar to the ones that were utilised to perform the test of the current work. Laboratory models of the same scale were tested by wind tunnel and by numeric simulations [8]. With the use of SST (shear stress transport) models in ANSYS CFX software which were able to replicate with remarkable accuracy, the drag force can be produced by their models in the wind tunnel. The models and boundary conditions with the best performance were replicated later by them in order to simulate the drag force produced by new geometries with added aerodynamic modifications aiming the drag force reduction. They reached up to a $70 \%$ of drag reduction with their more radical designs at high speeds. Other articles focused in buses' drag reduction by the utilization of CFD and wind tunnel design have been published [9-11]. A large amount of flow control techniques also have been developed and applied to buses scale model bodies. All of these researches have helped and will keep on helping us new aerodynamic solutions that could contribute to make more efficient energy saving land transports. The technology will never stop, which force us to use it to find new ways of designing our transports.

\section{Materials and Methods}

\subsection{Model Digitalization}

UAEM (Morelos State Autonomous University) has a small bus fleet for transportation of personnel and students. One of these buses (Fig. 1) was chosen as a starting point for the present study. This transport is Mercedes-Benz bus design, Boxer- 60 model made by

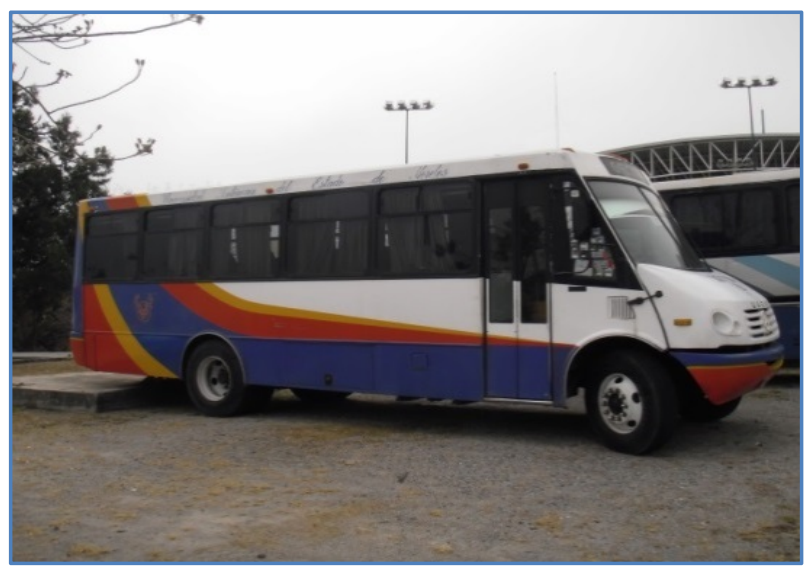

Fig. 1 Boxer-60 bus made by Marcopolo. 
Marcopolo. All of its measures were taken and were used to replicate a simplified 1:20 scale model in a CAD (computer-aided design) software.

Aiming to ease its reproduction and also to simplify numeric calculations, all the rounded edges and curved surfaces were replaced by straight edges and flat walls. The underbody that generally has holes and bumps was replaced by a flat surface. The tyres were considered without any kind of movements, and in general, all the shapes that were considered complex were simplified to avoid having zones that required high density meshing. Fig. 2 shows the final model printed with a thermoformable plastics $3 \mathrm{D}$ printer.

\subsection{Scale Model Manufacture}

Because of its dimensions, the model was separated into four parts to make them small enough to be inside the printing volume of the $3 \mathrm{D}$ printer which was used to manufacture them. The Cube $\mathrm{X}$ Trio sold by Cubify's is the 3D printer used to build the segments with a thermoformable PLA (polylactide) plastic. The thickness of the material of every layer added by the nozzle was configured to $0.25 \mathrm{~mm}$ per layer. When the four segments were finished, all of them were glued together and the surface was sanded to ensure an even and smooth surface without the small imperfections that the printing process leaves behind. Finally, the entire body was painted with lacquer paint to provide a uniform surface finish. The overall size of the model was of $150 \mathrm{~mm}$ tall, $130 \mathrm{~mm}$ wide and $472 \mathrm{~mm}$ long.

\subsection{Wind Tunnel Testing}

The printed model was attached to a one-component dynamometer. The used measuring device was a digital IMADA DS2-1 with a resolution of $0.001 \mathrm{~N}$ and an uncertainty under $1 \%$. The dynamometer was used to measure the component of drag force only parallel to the principal flow direction. This measuring arrange was installed in the new wind tunnel (Fig. 3) belonging to the CIICAp (Applied Science and Engineering Research Centre) from the UAEM. The overall dimension

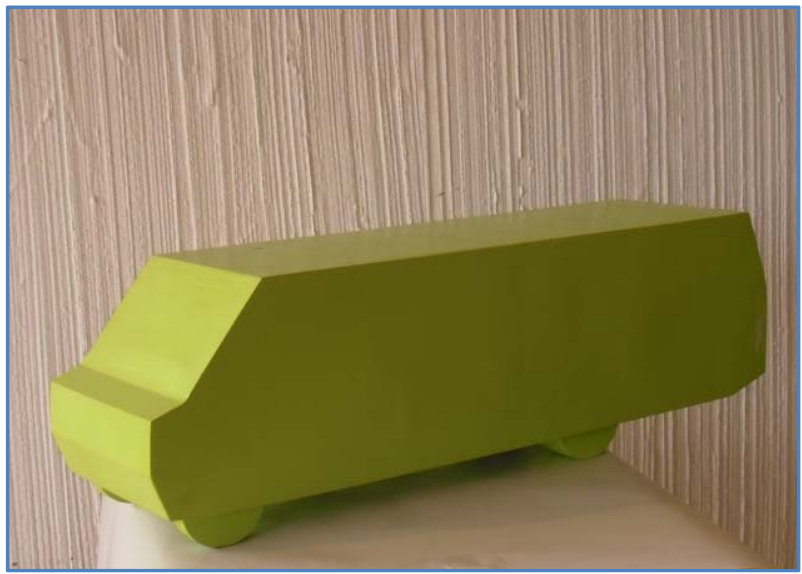

Fig. 2 Simplified bus model in a 1:20 scale.

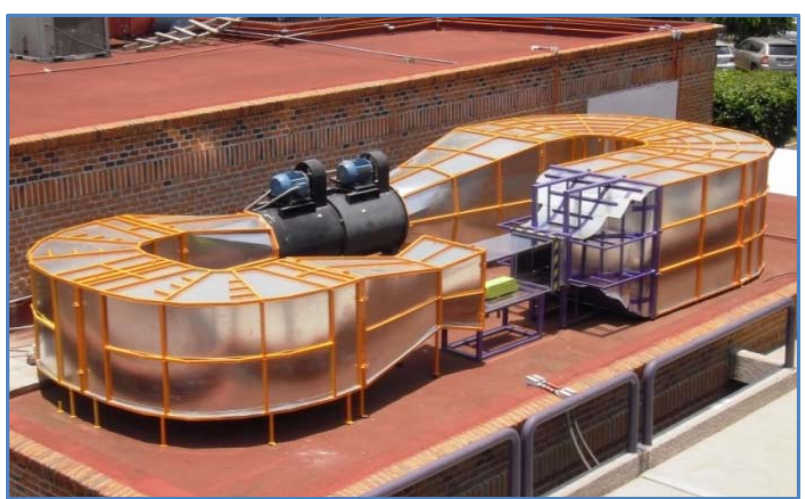

Fig. 3 Göttingen type wind tunnel in CIICAp.

Table 1 Fan operative conditions.

\begin{tabular}{ll}
\hline Spin frequency $(\mathrm{Hz})$ & Free stream air velocity $(\mathrm{m} / \mathrm{s})$ \\
\hline 34 & 19.2 \\
38 & 21.6 \\
42 & 23.7 \\
46 & 25.9 \\
50 & 28.0 \\
\hline
\end{tabular}

of the tunnel is of $9 \mathrm{~m}$ long, $4 \mathrm{~m}$ wide and $1.5 \mathrm{~m}$ tall. More specifically, the study zone is $1.8 \mathrm{~m}$ long, $0.5 \mathrm{~m}$ wide and $0.5 \mathrm{~m}$ tall.

The wind tunnel has two-axial fans connected one behind the other, every one of which is with a $7.5-\mathrm{kW}$ electric motor. Both of them are controlled by VFD (variable frequency drives) that allowed us to increase the rotor spin frequency with increments of $0.01 \mathrm{~Hz}$. The experimental arrange permitted free stream velocities of up to $35 \mathrm{~m} / \mathrm{s}$ with turbulence intensities under the $2 \%$, and it speeds up to $60 \mathrm{~m} / \mathrm{s}$ with turbulence intensities as high as $4 \%$. Table 1 shows the 
fan's rotor spin frequencies and the air free stream velocities measured inside the study zone. The air speed was measured with a Dwyer-471B digital anemometer, which can measure air velocities with increments of $0.01 \mathrm{~m} / \mathrm{s}$ with an accuracy of $3 \%$.

\subsection{Numerical Simulations}

With the academic version of the CAD software Solidworks, a 3D model of the CIICAp's wind tunnel study zone was created. Respecting the geometric dimensions of the scale model, it programmed a symmetry plane right in the middle plane parallel to the air stream flow. This decision was taken to save computational resources and simulation time in further simulation stages. As shown in Ref. [11], this decision will not cause an impact in the comparison between the experimental and numerical results because the shape of the body is not the one that could be consider extremely complex. To perform a proper comparison, it will be enough to multiply the simulated drag force by two. Once the geometry file was finished, it was exported to the academic version of ANSYS ICEM.

With the Solidworks Geometry, as a starting point, there were calculated three non-structured tetrahedral meshes. All of them with a prism configured on top of the surface of the bus body. The height of the first prism cell was configured to $0.00008 \mathrm{~m}$. After it, they were programmed 14 prism layers, more with a grow factor of $10 \%$. This decision was taken, thinking in assuring the best conditions for simulating the zone which was the fluid and the solid interact, especially in the zone where is documented the boundary layer separation. The difference between the three meshes was obtained by changing the maximum size of elements and by programing different elements grow rates after the prism. The first mesh had 1.8 million elements, the second had 2 million and the third had 2.2 million elements. After different preliminary tests, performed with the $k-k l-\omega$ turbulence model, and by comparing, these preliminary results with experimental data can be obtained by wind tunnel testing; It was determined that the performance of the second mesh was the most adequate, because the drag force predictions were fairly similar to the ones obtained by the third mesh but using less time for calculations. There, for the second mesh, was chosen to perform all the simulations required by the study with different turbulence models.

The chosen mesh was exported from ANSYS ICEM to the academic version of ANSYS FLUENT to replicate the experimental conditions with different turbulence models, in order to determinate which of the chosen ones could give the more accurate results. After looking at the literature and no finding a consensus, about which model to choose, it was decided to utilise four different turbulence models and different configurations of them to simulate the five free stream velocity conditions that were used during the wind tunnel testing. The turbulence models and the published studies they were used are: $k$-e RNG (Re-Normalisation Group) [12], $k$-e RNG with EWT (enhanced wall treatment) [3], $k-k l-\omega, k-\omega$ [13], $k-\omega$ SST [8] and RSM (Reynolds stress model) [14]. All of the simulations were performed using four processing cores in parallel. The pressure-velocity coupling scheme was configured as SIMPLEC, and the solver was a pressure based.

The boundary conditions for all cases were configured as follows: one velocity inlet with a $2 \%$ turbulence intensity and the same free stream velocity values, as shown in Table 1. Also in the exit, there was configured an outflow boundary. Side walls, the floor and the upper end of the domain were configured as non-slip walls.

The drag force from the simulations and the wind tunnel tests was used to calculate the drag coefficient changes between them dimensionless. The drag coefficient $\left(C_{d}\right)$ was calculated with the following equation:

$$
C_{d}=D /\left(\rho U^{2} A / 2\right)
$$

where:

$D$ is the drag force measured in the wind tunnel or 
calculated by CFD;

$\rho$ is the air density;

$U$ is the air free velocity;

$A$ is the frontal area of the bus scale model.

In order to study the numerical results in different ways, the upper side and underbody pressure coefficients profiles were exported from the symmetry plane in the contact zones between the fluid and the solid. All the results from the different turbulence models were plotted in the same figure to perform a dimensionless behaviour comparison. The pressure coefficient $\left(C_{p}\right)$ was calculated as following:

$$
C_{p}=\left(P-P_{r e f}\right) /\left(0.5 \rho U^{2}\right)
$$

where:

$P_{\text {ref }}$ is the static reference pressure measured in the upper side of the computational domain at the same distance of the inlet as the bus model;

$P$ is the dynamic pressure in the wall of the scale model;

$\rho$ is the air density;

$U$ is the air free velocity.

\section{Results}

After performing simulations replicating the five different experimental conditions using six turbulence models, all the resulting drag force values from the experiments and the numeric simulations were compared. Fig. 4 shows a comparative graph with the experimental and numeric drag coefficient values under different free stream velocities.

Table 2 shows the average relative error of drag coefficient values between experiments in wind tunnel and CFD simulations in ANSYS FLUENT.

Additional to drag coefficient comparisons, Figs. 5 and 6 show pressure coefficient graphs with dimensionless pressure values obtained from the underbody and the upper body right in edges from the symmetry plane along all the scale body's geometry, parallel to the free stream main direction.

\section{Discussions}

As it can be seen in Fig. 4 and as it was expected, all the models show similar tendencies but different magnitudes in the drag coefficient prediction. In general, the drag coefficient tends to be over-predicted when the air velocity increases, which means that the faster the stream is flown, the larger the relative error gets between the wind tunnel experiments and the numerical simulations, having this last ones bigger

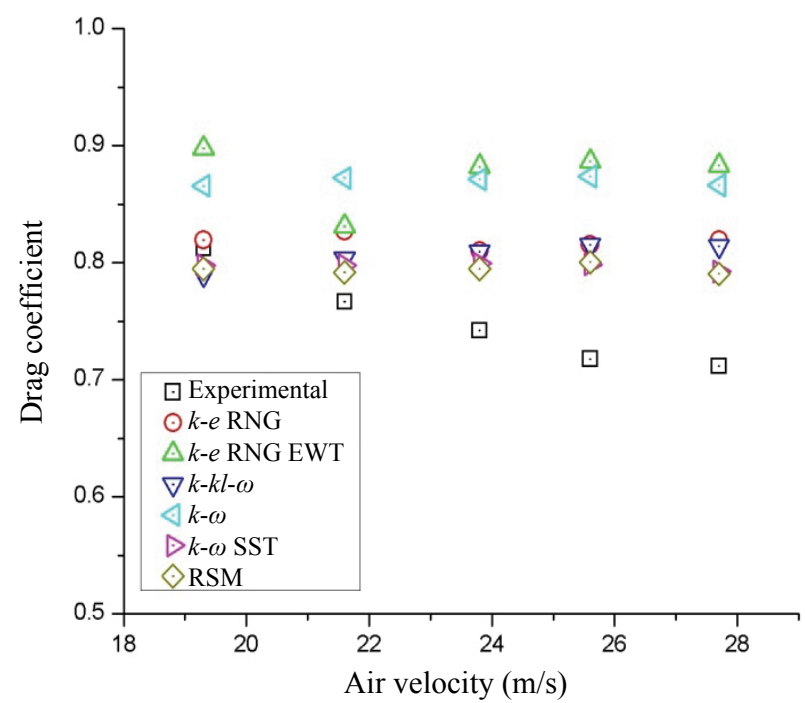

Fig. 4 Drag coefficient vs. different air velocities from experimental and numerical testing.

Table 2 Drag coefficient differences.

\begin{tabular}{lrrrrrr}
\hline \multirow{2}{*}{ Turbulence model } & \multicolumn{5}{c}{ Drag coefficient under different air velocity } & \multirow{2}{*}{ Average by model } \\
\cline { 2 - 6 } & $19.3 \mathrm{~m} / \mathrm{s}$ & $21.6 \mathrm{~m} / \mathrm{s}$ & $23.8 \mathrm{~m} / \mathrm{s}$ & $25.6 \mathrm{~m} / \mathrm{s}$ & $27.7 \mathrm{~m} / \mathrm{s}$ & \\
\hline$k$-e $\mathrm{RNG}$ & $0.80 \%$ & $7.84 \%$ & $9.17 \%$ & $13.55 \%$ & $15.06 \%$ & $9.28 \%$ \\
$k$ - RNG EWT & $10.45 \%$ & $8.38 \%$ & $18.88 \%$ & $23.50 \%$ & $24.06 \%$ & $17.05 \%$ \\
$k-k l-\omega$ & $2.82 \%$ & $4.79 \%$ & $9.05 \%$ & $13.54 \%$ & $14.40 \%$ & $8.92 \%$ \\
$k-\omega$ & $6.50 \%$ & $13.78 \%$ & $17.40 \%$ & $21.70 \%$ & $21.67 \%$ & $16.21 \%$ \\
$k-\omega$ SST & $1.87 \%$ & $4.02 \%$ & $7.70 \%$ & $11.21 \%$ & $11.32 \%$ & $7.22 \%$ \\
RSM & $2.18 \%$ & $3.24 \%$ & $7.08 \%$ & $11.50 \%$ & $11.04 \%$ & $7.01 \%$ \\
Average by free stream velocity & $4.10 \%$ & $7.01 \%$ & $11.54 \%$ & $15.83 \%$ & $16.26 \%$ & \\
\hline
\end{tabular}




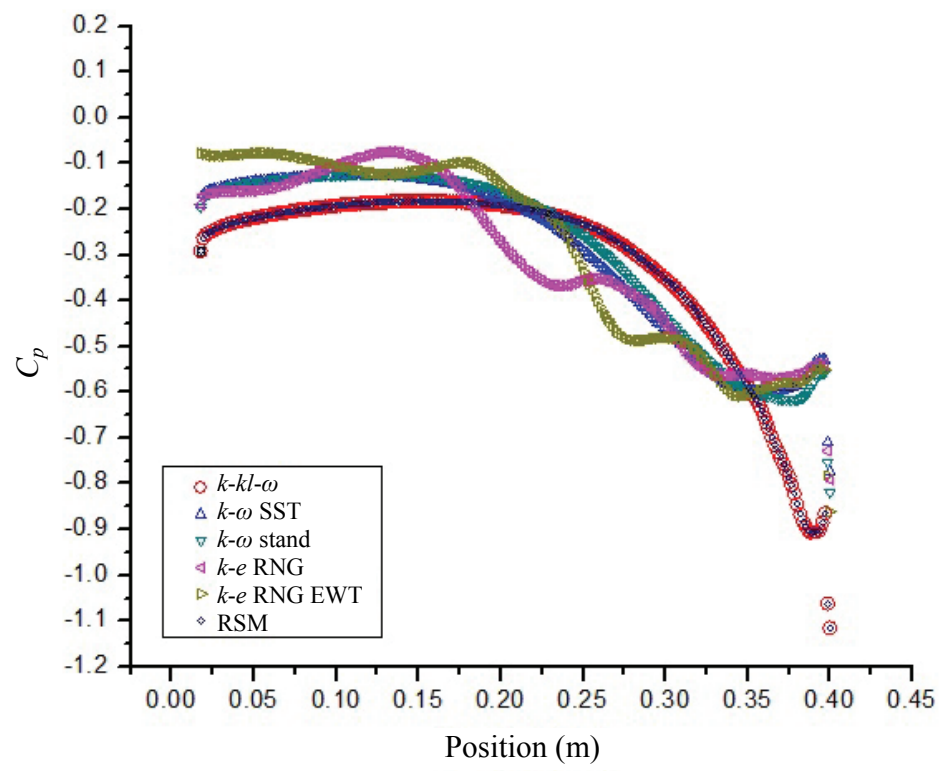

Fig. 5 Pressure coefficient graph from the symmetry plane in the superior edge of the body.

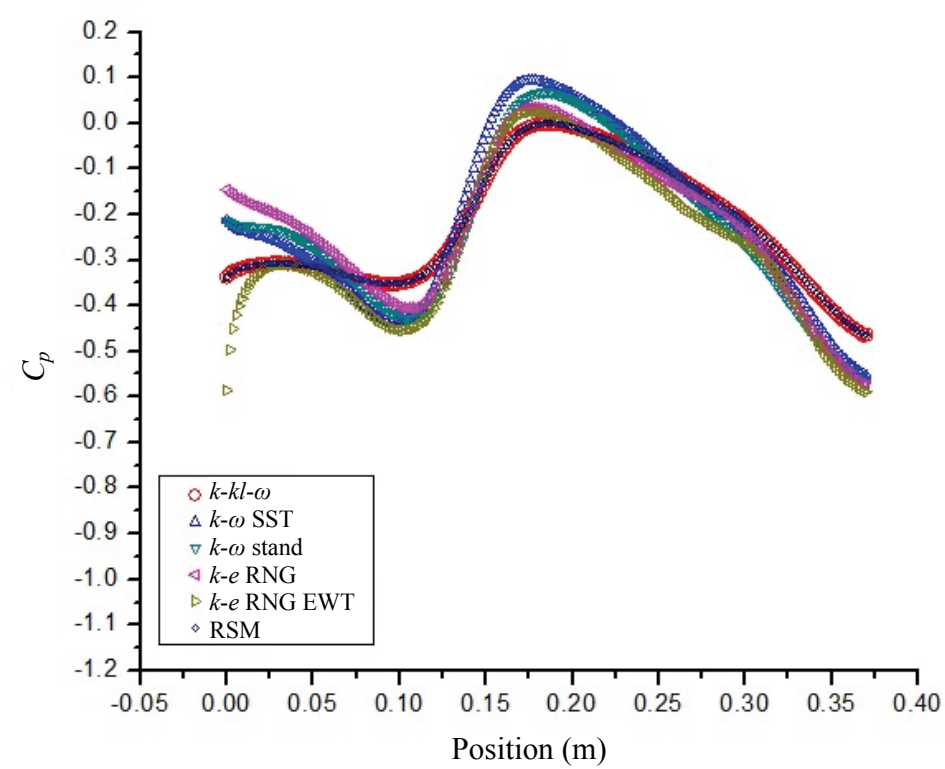

Fig. 6 Pressure coefficient graph from the symmetry plane in the inferior edge of the body.

values almost every time. This situation might be due to the increase of turbulence in the wake full of vortex that was not possible to study in this work because of the lack of equipment to visualize or characterize flow structures. With further studies, applying techniques like the PIV (particle image velocimetry), LDV (laser Doppler velocimetry), it could also be determinated which turbulence models are more capable of predicting the formation of vortexes in the wake, at the same time, they predict the drag force produced by the interaction between the body and the fluid.

In average, the simulations under $19.3 \mathrm{~m} / \mathrm{s}$ air speed had a relative error of $4.1 \%$, and by augmenting the air speed up to $27.7 \mathrm{~m} / \mathrm{s}$, the relative error between the simulations and the experiments reached an average of $16.26 \%$. The smaller errors were achieved with the RSM model, predicting the drag coefficient with average of $7.01 \%$, while the $k$-e RNG EWT got the 
worst results averaging $17.05 \%$ of relative error. In all cases, the models $k$-e RNG, $k-k l-\omega, k-\omega$ SST and RSM gave excellent results. Being the last two, the ones predicted the drag force more accurately. The big difference among them was the simulation time that they required to predict converging solutions. The $k-\omega$ SST model had a slightly larger error than the RSM by $0.021 \%$, but the results convergence came in average $30 \%$ faster than it with the other turbulence model.

The pressure coefficient graphs confirm the vital need of measuring the pressure field distribution across the scale model surface with pressure tabs. This is because it is vital to know the force more accurately that the flow transfers to the body. In the $C_{p}$ graph from the upper edge of the body, as well as in the under edge graph, it can be seen that the $k-k l-\omega$ and RSM have extremely similar behaviours. The relative error difference between these two models in the $C_{p}$ graphs is of only $1.01 \%$, but the computational time required by the RSM model was up to $20 \%$ higher that the $k-k l-\omega$. This numbers probe that the use of a model, , as robust as the RSM to achieve acceptable results, is not always necessary. But, it is recommendable again to validate these simulations with pressure tabs measurements. The pressure coefficient predicted by the $k-k l-\omega$ model is higher to the predicted by the RSM in the front, but as the graph advance is given towards the middle zone of the model, the values gradually get closer. From that point, the pressure predicted by the RSM is higher until the graph reaches a zone in the back where this model calculates a much more drastic pressure drop than the $k$ - $\omega$ SST, possibly because an over prediction of the suction effect caused by the boundary layer separation begins some centimetres before the end of the body. Looking at the inferior $C_{p}$ graph, we can see that the three models that we have mentioned more predicted similar behaviours but the $k-\omega$ SST shows a more extremist than the RSM. This model gets the higher pressure values in the positive side of the graph, as well as $13 \%$ lower values in the last part of the graph.

\section{Conclusions}

The present work has presented a series of tests, experimental ones, as well as numerical of simplified scale bus models, aiming to determinate from a group of turbulence models which can predict the aerodynamic drag produced in the wind tunnel more accurately. This is to perform new modifications on the bus bodies and to make them more efficient and aerodynamic.

After conducting wind tunnel testing under five different free stream velocities, the same experimental conditions were replicated in ANSYS FLUENT with six different turbulence models. The results from all the tests were compared, obtaining the best results with the RSM model, which averaged a relative error of $7.01 \%$ compared whit the experiments. Despite the similarity between experimental and CFD results, the $k-\omega$ SST model, a model far less complicated than the RSM, is the one that we recommend to be using in future simulations because it required computational times $30 \%$ shorter than the RSM. Considering that it gains differences of $7.22 \%$ against wind tunnel testing utilising much lower computational resources, the $k-\omega$ SST model is shown to be the best option to simulate new geometries replicating the wind tunnel conditions and saving precious time and monetary resources in long and expensive wind tunnel tests. So, in the future, it is to be expected for our group to continue with the work and develop new aerodynamic bus designs using the knowledge acquired in this stage of our research.

\section{References}

[1] Ahmed, S. R., Ramm, G., and Faltin, G. 1984. "Some Salient Features of the Time-Averaged Ground Vehicle Wake." SAE Technical Paper No. 840300. SAE International. Accessed May 20, 2013. http://papers.sae.org/840300/.

[2] Vino, G., Watkins, S., Mousley, P., Watmuff, J., and Prasad, S. 2005. "Flow Structures in the Near-Wake of the Ahmed Model.” J. Fluids Struct. 20 (5): 673-95.

[3] Bruneau, C., Creusé, E., Gilliéron, P., and Mortazavi, I. 2014. "Effect of the Vortex Dynamics on the Drag Coefficient of a Square Back Ahmed Body: Application to 
the Flow Control." European Journal of Mechanics B/Fluids 45: 1-11.

[4] Fares, E. 2006. "Unsteady Flow Simulation of the Ahmed Reference Body Using a Lattice Boltzmann Approach." Comput. Fluids 35 (8-9): 940-50.

[5] Thacker, A., Aubrun, S., Leroy, A., and Devinant, P. 2012. "Effects of Suppressing the 3D Separation on the Rear Slant on the Flow Structures around an Ahmed Body," J. Wind Eng. Ind. Aerodyn. 107-108: 237-43.

[6] Serre, E., Minguez, M., Pasquetti, R., Guilmineau, E., Deng, G. B., Kornhaas, M., Schäfer, M., Fröhlich, J., Hinterberger, C., and Rodi, W. 2013. "On Simulating the Turbulent Flow around the Ahmed Body: A French-German Collaborative Evaluation of LES and DES." Comput. Fluids 78: 10-23.

[7] François, D. G., Delnero, J. S., Colman, J., Marañón, D. L. J., and Camocardi, M. E. 2009. "Experimental Determination of Stationary Aerodynamics Loads on a Double Deck Bus." Presented at 11th Americas Conference on Wind Engineering, San Juan, Puerto Rico.

[8] Sharma, R., Chadwick, D., and Haines, J. 2008. “Aerodynamics of an Intercity Bus." Wind and Structures 11 (4): 257-73.
[9] Pranami, K. G., Shah, D. V., and Patel, B. T. 2014. "Improvement of Bus Body Aerodynamic Shape by CFD Analysis." International Journal for Scientific Research and Development 2 (3): 1698-703.

[10] Raveendran, A., Sridhara, S. N., Rakesh, D., and Shankapal, S. R. 2009. "Exterior Styling of an Intercity Transport Bus for Improved Aerodynamic Performance." SAE International 8 (2): 9-16.

[11] Saxena, M. R., Vishwakarma, Y. K., Upadhyay, S., and Nagarajan, P. K. 2014. "Aerodynamic Improvement of Bus Body by Using CFD." International Journal of Applied Engineering Research 9 (4): 435-43.

[12] Muthuvel, A., Murthi, M. K., Sachin, N. P., Koshy, V. M., Sakthi, S., and Selvakumar, E. 2013. "Aerodynamic Exterior Body Design of Bus." International Journal of Scientific \& Engineering Research 4 (7): 2453-7.

[13] Khalighi, B., Jindal, S., and Iaccarino, G. 2012. "Aerodynamic Flow around a Sport Utility Vehicle-Computational and Experimental Investigation." J. Wind Eng. Ind. Aerodyn. 107-108: 140-8.

[14] Meslem, A., Bode, F., Croitoru, C., and Nastase, I. 2014. "Comparison of Turbulence Models in Simulating Jet Flow from a Cross-Shaped Orifice." Eur. J. Mech. B/Fluids 44: 100-20. 